\title{
DoE provides funds for human genome sequencing
}

San Francisco

As the debate rages in Washington over what should be done about mapping and sequencing the human genome, who should do it and how it should be done, the US Department of Energy (DoE) has given three of its national laboratories a free hand - and some money (see p. 101) - for finding technologies to carry out the project.

Last year, DoE forced the pace by naming Lawrence Berkeley and Los Alamos National Laboratories as human genome centres. That move may have been premature, says Dave Smith, currently director of DoE's efforts, but work will not necessarily be limited to these facilities in the future.

Los Alamos, building on its GenBank experience in computing and datahandling, is studying methods for storage and distribution of the vast amounts of data to be generated by the project. Experiments are also under way on computer models of genome organization in order to predict the barriers to mapping and sequencing presented by the large amount of repetitive DNA in the human genome. A robotics project aims to automate some of the tedious jobs involved in DNA and sample preparation.

The choice of Lawrence Berkeley Laboratory as a centre for genome work came about partly because of its proximity to the University of California campus at Berkeley. Scientists at Lawrence Livermore Laboratory, some 30 miles away, were surprised that they too were not granted official status as a centre, but expect to be closely involved in the project.

Livermore has been in the field for five years, through its development of flow cytometry for sorting human chromosomes, and the resulting National Gene Library Project, which has generated small-insert phage libraries for each human chromosome and has now begun the next phase: large-insert phage and cosmid libraries that will be vital to the mapping effort.

All three laboratories are considering the newly available mapping approaches that use the rare-cutting restriction enzyme, Not I to generate DNA fragments of up to 1,000 kilobases in size, which can then be fractionated on pulsed-field gels. Scientists at Berkeley are concentrating their efforts on Yeast Artificial Chromosome (YAC) vectors, which have the capacity to accommodate the NotI fragments. Worries about difficulties in cloning fragments in YAC vectors led the Livermore team to attempt an alternative approach, in which the 100 or so Not I fragments per chromosome are ordered through the use of 'linking probes' which span NotI cleavage sites. Specialized vectors for the selective cloning of such probes are being developed at Livermore.

Los Alamos and Livermore scientists have begun their mapping efforts in earnest, starting with chromosomes 19 at Livermore, and 16 at Los Alamos, both chosen for their size and concentration of clinically relevant markers, including the DNA repair genes, in which both laboratories have research interests.

The Livermore team is using computer modelling to optimize the DNA fingerprinting method, which determines clone overlap through a comparison of restriction patterns. Using fluorescently labelled DNA, enzymes that generate $80-100$ fragments per cosmid, and an Applied Biosystems automated sequencer to read and store the data from the gels, project director Tony Carrano estimates that the team could map chromosome 19 to nearcompletion in 110 gel-days, a project they will begin in a month or so.

Los Alamos scientists have their eyes on the so-called 'random probes' method, proposed by Hans Lehrach, now at the Imperial Cancer Research Fund in London. This is a gel-free technique in which random oligomers are hybridized to arrays of thousands of DNA spots, or possibly even bacterial colonies, on filters. A computer program would then use the hybridization data to order the clones. Despite the attractiveness of a gel-free system that might not even require DNA preparation, the method is unproven, with many technical hurdles yet to surmount. In the meantime, the Los Alamos team has begun limited mapping of chromosome 16 by working with phage clones, starting with clones that have already been genetically mapped to sites on the chromosome.

Beyond the genome-mapping phase, which most expect to complete within 3-5 years, looms the arduous task of sequencing. Lawrence Berkeley scientists are counting on automated advances in current sequencing technology, such as those being developed by the Japanese, to bring the cost down tenfold from the current $\$ 1$ per nucleotide. Los Alamos scientists are gambling on an effort to develop gel-free DNA sequencing using a flow system in which a single fluorescently labelled DNA molecule would be bound to a matrix, progressively digested with endonuclease, and the bases identified by flow cytometry. Although early results are promising, the project is a long-shot, says Ed Hildebrand, coordinator of the genome effort at Los Alamos, but one worth risking.
Sex test banned

New Delhi

Maharashtra has become the first state in India to ban prenatal sex determination testing on pregnant women. Chief Minister S.B. Chavan said the decision was taken because of "deep concern" over the widespread abuse of the test for aborting female fetuses. Legislation is to be introduced in February.

The ban applies only to private hospitals and laboratories. Government-run hospitals and research institutions of the Indian Council of Medical Research can carry out the test provided the woman is over 35 years of age and already has a child with an inherited defect.

Selective abortion of females has long been the subject of controversy in India and Maharashtra's action follows complaints from women's groups. Although gynaecologists are divided over the wisdom of a blanket ban on private practitioners, it is likely that more states will follow Maharashtra's.

K.S.J.

\section{Heineken award \\ London}

Thomas R. Cech, of the University of Colorado, Boulder, is to receive the 1988 Dr H.P. Heineken Award for his discovery that RNA can act as an enzyme. The award, worth Dfl250,000 $(\$ 130,000)$ is the largest Dutch prize in cash for scientific research. It is awarded every two years by the Heineken Foundation on the nomination of the Royal Netherlands Academy of Arts and Sciences. Cech's breakthrough, made in 1986, raises new questions about molecular evolution, with the possibility that RNA was the primordial prebiotic molecule and that proteins and DNA evolved subsequently.

S.L.H.

\section{US research}

\section{Washington}

THE latest figures compiled by the National Science Foundation (NSF) show that Johns Hopkins University is still the largest recipient of federal research dollars, largely on the strength of money allocated to the Applied Physics Laboratory for defencerelated research. The NSF data are for the 1986 fiscal year. The government committed $\$ 6,538$ million for research and development to all US universities and colleges, of which 85.5 per cent went to the top 100 institutions, and 25.9 per cent went to the top ten.

J.P.

Johns Hopkins University

$\$ 000$

(188,120

Stanford University

University of Washington

180,186

University of California, San Diego $\quad 133,243$

Columbia University, main division $\quad 127,131$

University of California, Los Angeles $\quad 125,483$

University of Wisconsin, Madison $\quad \mathbf{1 2 0 , 6 2 6}$

Cornell University

112,707

Yale University 